\title{
THE PATHOLOGY OF IDIOPATHIC DETACHMENT OF THE RETINA.
}

\author{
By Arthur H. H. Sinclair, M.D., F.R.C.S.Ed. \\ From the Laboratory of the Royal College of Physicians, Edinburgh.
}

(Plate XVII.)

\section{Work of Previous Investigators.}

VON GRAEFE originally regarded subretinal hæmorrhage as the cause of detachment, but this view was soon found to be erroneous. The theory of choroidal transudation next held the field for some years, but the sudden occurrence of more or less extensive detachment, in eyes of subnormal intraocular tension, made it evident that some other explanation must be sought. The mechanical theory, on which it was put forward that the elongation of the eyeball which takes place in myopia, caused detachment of the retina, has also had its supporters. The theory of "vitreous fibrillation and contraction" and that of "diffusion" are the most recent and important, and require some detailed consideration.

The theory of vitreous contraction and fibrillation, though also supported by other observers, has received its real prominence from Professor Leber, who, in a lecture delivered before the Ophthalmological Assembly at Heidelberg in 1882, expressed his views in favour of this theory; and of Nordenson, who followed up the investigations begun by Leber, and specially devoted himself to the study of the morbid anatomy of the vitreous body from the histological point of view.

He describes the changes which take place in the vitreous body as follows :-

There is increase of the tissue elements of the vitreous. The fine fibrillæ which enter into the normal structure of the vitreous become thickened and tortuous, the cell elements increase in number, and the vitreous, as a whole, enters upon a progressive process of shrinkage. If the case be one in which the vitrio-retinal attachments are normal, the progressive shrinkage results later in a complete funnel-shaped detachment of the vitreous body. The connection at the optic nerve remaining intact forms the narrow end of the funnel, and the normally more adherent zone at the equator forms the expanded end. The space left between the retina and the retracting hyaloid membrane, coincidently with the vitreous shrinkage, fills with fluid. 
Is the shrinkage proceeds still further, traction upon the retina takes place at the parts where the attachments are still intact, namely, at the papilla and equator. It may happen that the retina gives way where it is dragged upon at the equator; in such a case rupture admits the subhyaloid Huid to the posterior surface of the retina, and so detachment is rapidly protucel.

The course of events as above described is, however, according to Nordeuson, subject to modifications.

First, the retina may rupture within the area of hyaloid adhesion, and so he protected from the entrance of the subhyaloid fluid by way of the rupture. 'The detachment of the retina would then proceed slowly from vitreous traction, and from such fluid as might accumulate behind it from the vitreous and (in vacuo) from the choroid.

Siecomlly, the vitreous body may not become separated from the retina, as stated in the first-mentioned class of cases, but may exercise traction from the beginning upon the posterior part of the retina, and so produce detachment, primarily by the collection of fluid behind the retina from the vitreous or (in vacioo) from the choroid. The retinal rupture occurs in each case from vitreous traction as before, but it is secondary to the formation of detachment.

Lastly, it is not denied that such forms of detachment as might be produced by a subretinal effusion of fluid may sometimes take place in rare cases it in albuminuric retinitis.

By the Leber-Nordenson hypothesis we are able to understand how the retina may be extensively detached in a very short time without increasing the intraocular tension. The free communication -by way of the rupture - of the fluid lying in front with that lying behind the retina also affords explanation of the occurrence of sudden spontaneous cure.

Tordenson states that in all his investigations in this connection he found pathological conditions of the choroid present. He regards disturbances of nutrition, particularly those produced by inflammatory processes affecting the choroid, as the cause of the changes which take place in the vitresus. $\mathrm{He}$ states that the same marked changes of the vitreous have been caused by intraocular tumours, and have also been found in eyes which have been affected by chronic glaucoma.

The theory of diffusion, which was originated and supported by Raehlmann of Dorpat, is explained by him as follows:-

The retina is compared to the animal membrane of a dialyser, which has on one side a saline solution represented by the vitreous humour, and on the other an albuminous solution formed by choroidal transudation. $\mathrm{He}$ regards the old basis of choroidal transudation as the starting-point, which sets up the altered relationship of osmotic pressure, and causes a pathological process of diffusion to begin. The fluid of the vitreous being almost entirely saline, having crystalloid properties, diffuses readily through the retina, and thus fluid may be collected behind the retina without increase of intraocular pressure.

Raehlmann farther points out, in support of his theory, the marked differences in colour, in the amount of albumin contained, and in specific gravity, which he has found to exist between the post-retinal fluid, and that of the vitreous. In several instances in which retinal rupture could not be discovered, Raehlmann aspirated the subretinal fluid. 
The aspiration was performed under ophthalmoscopic control, by means of the Pravaz syringe. The fluid so removed was found to be of a greenishyellow colour. The area of the detachment was observed to change colour on the removal of the fluid, losing its greenish or blue hue, and becoming more like the normal fundus. In some instances the amount of albumin was estimated by Scherl, ${ }^{1}$ and was found to equal, and sometimes to exceed, that of serous effusions. The well-known tendency which a partial detachment, at the upper part of the fundus, has to extend downwards, is referred to by Raehlmann as the "phenomenon of sinking," and is ascribed by him to the relatively high specific gravity of the post-retinal fluid, as compared with that of the vitreous. Raehlmann regards retinal rupture as secondary to detachment, and believes it to be the result of the pressure of post-retinal fluid.

Rupture of the detached retina is observed ophthalmoscopically in a certain proportion of cases, variously estimated by different observers.

Nordenson, from 119 cases, places its frequency at 38 per cent., while Galezowski² puts it at 20 per cent., from the examination of 649 cases of detachment. Galezowski regards the vicinity of the ora serrata as the most frequent site.

The pathology of rupture has already been touched upon in giving the views of Leber and Nordenson and of Raehlmann.

Collins ${ }^{3}$ describes two cases which were examined microscopically by him. In each case a patch of intimately adherent much degenerated retinal tissue was found attached to the choroid, with a corresponding gap in the detached retina. Elsching in 1892 brought forward this theory, ${ }^{4}$ having observed two cases in which rupture had occurred, where, from old inflammatory adhesion, the retina and choroid were united.

While acting as clinical assistant at the Royal London Ophthalmic Hospital I saw a case similar to the above, under the care of Mr. Lang. In this case detachment had taken place in an eye in which a small round patch of central choroiditis was present, with the result that a small rounded aperture could be seen in the detached retina, through which, with the appropriate lens, the adherent macular portion could be seen. It has often been observed clinically that the torn margins of the rupture are turned towards the anterior or vitreous surface. Various reasons have been given to account for this.

Leber regards the attraction exercised by the strands of contracting vitreous fibrillæ as the cause, while Raehlmann would ascribe it to the pressure of the post-retinal fluid at the time when rupture occurred.

In several instances $I$ have dissected the retina of the rabbit (post-mortem) from its intraocular attachments, and have examined it floating in water. Under these conditions, the tendency of the cut

${ }^{1}$ Arch. f. Augenh., Wiesb., 1893, Bd. xxvii. S. 26.

2 Rec. d'ophth., Paris, 1883, Sér. 3, tome v. p. 669.

${ }^{3}$ Trans. Ophth. Soc. U. Kingdom, London, 1896, vol. xvi. pp. 83-\$4.

4 Klin. Monatsbl.f. Augenh., Stuttgart, 1892, Bd. xxx. S. 416.

20—JL. OP PATH. - VoL. VII. 
edges to turn forward towards the vitreous surface was still well marked, and could only be explained by the natural tissue resilience of the retina.

\section{Experiments in the Artificial Production of Detachment.}

I will now give a short account of some of the points of interest in connection with my experiments.

The animals employed were rabbits. At the time of operation each animal was thoroughly anæsthetised and suitably placed in the prone position. The operations were carried out under ophthalmoscopic control. The direct method was used in following the procedure with the ophthalmoscope, so that one hand remained free to guide the instrument used. A sufficient dilatation of the pupil was always obtained before the anæsthetic was given. The most careful antiseptic measures, sterilisation of instruments and materials used, were carried out. The instruments were in some instances modified, or specially devised for use.

The normal fundus of the rabbit is in some respects peculiar. The optic nerve enters the eyeball above and to the temporal side of the posterior pole. The nerve fibres on entering the eye retain their myelin sheath, and are distributed so as to form an opaque band across the fundus. This opaque band of fibres forms an angle of $30^{\circ}$ with the horizontal, having the nasal extremity higher than the temporal, and forming a brush-like margin where the fibres lose their sheaths and are distributed to the general fundus. The subhyaloid vessels are seen coursing upon the opaque band of fibres. Plate XVII. Fig. 1, from a photograph of the dissected retina, shows the appearance of these fibres.

\section{Copper in the Vitreous.}

The introduction of particles of sterilised copper into the vitreous was performed by means of the needle of a hypodermic syringe, which was first charged with the desired amount of filings, introduced through the sclera and cleared by a wire previously fitted.

EXPERIMENT 1.- In this case a small mass of copper filings was successfully introduced into the centre of the vitreous and was well seen with the ophthalmoscope. On the second day a pearly white opacity of the vitreous had formed between and immediately around the particles of copper, which still remained aggregated together. The opacity increased from day to day between and around the particles. On the fifth day some of the copper had changed its position; this was followed by the dense whitening of the vitreous. On the eighth day streaks of opacity were seen extending into the vitreous in various directions. These changes in the opacity of the vitreous increased steadily from day to day. On the twenty-fifth day the greater part of the fundus was obscured from view. Detachment of the retina could be seen. The formation of new blood vessels was observable. On the fortieth day the cornea had become so opaque that no farther ophthalmoscopic examination could be made.

On post-mortem examination of the eye it was found to have shrunk to. one-third of the normal size, and to be filled with fibro-cellular tissue. 
EXPERIMENT 2.-In this case the same procedure was carried out as in Experiment 1. On the second day the mass of filings had sunk to a slightly lower position than when first observed, and was surrounded by the same pearly opacity of the vitreous as before described. The subhyaloid vessels were engorged. There was a large hæmorrhage of the retina at the upper part of the fundus. On the fourth, fifth, and sixth days the opacity had steadily increased in extent; there was also some general haziness. On the tenth day the white opacity was in contact with the retina at the lower part of the fundus. The vitreous on each side of the opacity had become quite clear. There was a small bubble of gas at one side close to the copper filings.

During the following two weeks the opacity increased, the gas bubble was absorbed, and some new blood vessels began to appear on the opaque vitreous. By the fortieth day extensive detachment of retina could be seen, the opacity being much increased, and the whole vitreous hazy. Ilate XVII. Figs. 2 and 3, from photographs of sections of the eyeball show some of the micrescopic appearances.

\section{Howorrhage into the Vitreous.}

EXPERIMENT 3.-By the use of a large flat needle, extensive hæmorrhage was produced at the upper part of the fundus. On the third day extensive hæmorrhage had taken place into the vitreous, so that the fundus could not be seen. At the end of the third week the vitreous was sufficiently clear at parts to give some view of the fundus, when detachment of the retina was seen. As the vitreous cleared, extensive detachment could be seen, which increased until the thirty-ninth day, beyond which the observations were not carried.

\section{The Artificial Production of Detachment by the Injection of Fluid behind the Retina.}

In performing this operation, under ophthalmoscopic control, it was found advantageous to be able to command the movements of the eyeball, so that when placed in any desired position it could be so maintained without employing the hand of the operator to hold it. For this purpose I had a pair of forceps made with detachable blades, which were applied separately to the eyeball from each side, and afterwards locked. The pressure could be regulated by a spring and screw. The handles which were directed downwards could be supported by a pad in any position required.

The subretinal injection was carried out with a Liston's syringe, slightly modified, and supplied with elastic bands.

The injection of normal salt solution was performed as follows:-

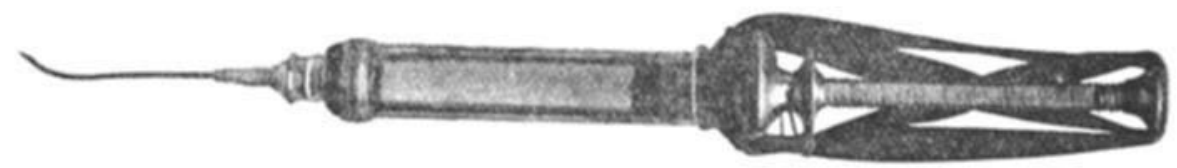

FIG. 1.

The syringe was half filled with the solution. The screw upon the piston rod was then advanced up to the end of the barrel of the syringe. The elastic bands attached to the body of the syringe were stretched over the end of the piston rod. 
From a syringe thus prepared for use, the post-retinal injection can be regulated in amount and in rate of introduction, and all jerling of the piston can be avoided. The retina being readily ruptured by too sudden or forcible injection it is necessary to avoid such, and this can be successfully accomplished by the method described.

The operation of subretinal injection is performed by making an oblique puncture through the sclera, a little behind the tendon of the superior rectus. The puncture is made with a Graefe's knife, penetrating the sclera very obliquely from before backwards, and is of such size as to admit the needle, without leaving any space round it through which the injected fluid could regurgitate. At the time of operation a second puncture is made into the vitreous, so as to allow some fluid to escape. This prevents an undue rise in the ocular tension.

On introducing the needle care is taken to keep it behind the retina throughout. When the needle has reached a point beyond the middle of the fundus oculi, so that the chances of regurgitation are reduced to a minimum, the screw on the piston rod is slowly retracted, and the injection then carried out by elastic pressure on the piston.

Experiment 4.-By the above method a large detachment was produced under ophthalmoscopic control. The animal was killed at once, before recovering from the anæsthetic. The eye was frozen, and the cut surface showing the detached retina was photographed (Plate XVII. Fig. 4).

EXPERIMENT 5.-By the same method about 5 minims of salt solution was introduced behind the retina. The retina was seen to change its position as the injection was made. No rupture could be detected ophthalmoscopically. On the eighth day extensive detachment was observed. On the twelfth day some parts of the detachment had beconse adherent to the choroid. On the twenty-eighth day the entire retina had become reattached. The characteristic pigmentary changes were seen over the reattached area.

EXPERIMENT 6.- In this case, by a slight modification of the procedure, a small quantity of the vitreous was taken up by the syringe and injected behind the retina. A detachment of considerable extent was produced at the lower part of the fundus. This detachment was kept under observation, and was seen to have completely healed by the end of the sixth week.

In the following experiments, in addition to primarily establishing cletachment by the method already described, an attempt was made to estimate the effect of replacing the post-retinal fluid with a solution containing active fibrinogen.

The idea of estimating the effect of fibrinogen as a therapeutic agent in this connection was suggested to me by Mr. George Berry, and I have to thank him, not only for his suggestion, but also for the encouraging interest which he has taken in my experiments.

The difficulties entailed in the process of separating from the blood a proteid substance such as fibrinogen, and the insoluble nature of that substance when obtained, together with the necessity for 
preserving it in a sterile form, suggested the advisability of preserving blood plasma, and using this substance for my purpose.

The following is the way in which blood plasma was preserved for use :-

In a clean 2-litre glass jar was placed 80 c.c. of a 2 per cent. oxalate of potash solution. The jar was then covered and sterilised in the usual way, and kept ready for use. When a convenient opportunity arose, 2 litres of blood, drawn directly from the jugular vein of a horse into the jar, were obtained. After sufficient time had been allowed for the sinking of the blood cells, the plasma was conducted into sterilised flasks, and so kept ready for use. Small quantities were also preserved in sealed glass tubes, 4 c.c. in each.

My idea was to introduce into the post-retinal space fluid blood plasma, which would soon after introduction form a clot, the contraction and organisation of which might, it was hoped, help to fix the retina in its normal position. I bad next to arrange means by which coagulation could be obtained at will, and to ascertain the time required for its completion.

By a series of experiments I found that 0.03 of 1 c.c. of a 20 per cent. solution of calcium chloride, added to 4 c.c. of blood plasma, caused a firm clot to form, in from twelve to fifteen minutes at the body temperature. This proportion of calcium chloride solution, when carefully mixed with the plasma, neutralised the potassium oxalate, and so brought about coagulation as desired.

The coagulum thus produced was firm and tough, of opaque amber colour, and contracted with the effusion of serum in the ordinary way. The sterility of the plasma was frequently tested by repeatedly placing small quantities in the incubator, and keeping each specimen under observation for sufficient time to ensure its sterility.

In order to carry out the removal of the post-retinal fluid, and to replace it by coagulating blood plasma at one operation, I had the following apparatus made :-

Two small-sized hypodermic syringes--of Liston's pattern-were attached side by side by spring catches to a single metal plate. One syringe, with elastic over the piston, was used for injecting the plasma, while the other was used for aspirating the subretinal fluid.

A double channelled $\mathrm{Y}$-shaped needle with small detachable rubber-tubing connections, fitted with clips, completed the apparatus (Fig. 2).

The operation of replacing the post-retinal fluid with coagulating blood plasma was performed, with the help of an assistant, as follows :-

The site of detachment was located, and a corresponding area of sclera exposed. The needle with attached tubing was filled with normal salt solution, and the small clips placed in position, to prevent the escape of fluid during manipulation. The needle was then introduced through the sclera into the post-retinal space, and directed by use of the ophthalmoscope to the part where detachment was most marked. 
The assistant having mixed the calcium chloride with the plasma, and filled the injecting syringe, the needle was connected with the syringes. The clip was then removed from the tube of the aspirat-

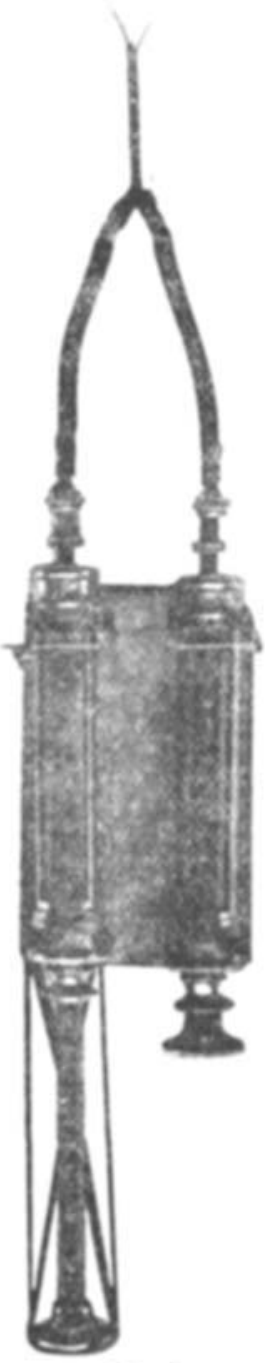
ing syringe, and a small quantity of post-retinal fluid withdrawn by advancing the screw on the piston rod. The clip on the injecting syringe was then removed, and by relaxing the screw on the piston rod, to the same extent as the other had been advanced, the elastic pressure slowly effected the injection.

Thus the amount of post-retinal fluid withdrawn was exactly replaced by coagulating plasma.

EXPERIMENT 7.-Artificial detachment was produced, as before described, and kept under observation for eighteen days. During the observations no rupture of the retina was detected. On the eighteenth day the postretinal fluil was replaced by coagulating plasma. After the injection of the plasma no retinal rupture could be seen. On the sixth day a vertical cleft was observed in the retina. Through this cleft a mass of yellow fibrin could be seen presenting. The retina on either side of the cleft was straw-coloured, from the colour of the fibrin behind it. Eight days after its introduction the mass of fibrin had come througl the retina, and lay in the vitreous.

EXPERIMENT 8.-A suitable artificial detachment having been produced, coagulating plasma was introduced behind the retina, as described. On the second day the detachment had a more irregular surface, and was somewhat yellow. On the third day there was no change. On the fourth day the fibrin was very clearly seen through the retina. The general fundus did not show any change. On the fifth day the tibrin was seen through the retina as before. The greneral fundus was reddened with the formation of greenish striæ upon it, which appeared like extending detachment. On the sixth day detachment had extended over a large part of the fundus. A bright red inflanmatory appearance characterised the undetached parts of the retina. On the seventh day detachment still increased, and redness of fundus decreased. On the eightl day detachment further increased, and undetached portions had nearly returned to normal colour. On the twelfth day the undetached portions had the normal appearances. The

FIG. 2.-Showing syr-detachment was much reduced, but still present at the inges as arranged for upper part, and, on the temporal and nasal sides, towards replacing the post- the periphery. Further ohservations showed the comretinal fluid with blood plete disappearance of all inflammatory reaction, but the plasma. persistence of detachment. No retinal rupture was discovered in this case.

Experiment 9.-The artificial detachment was produced as before. In order to estimate the effect of a less active preparation, the blood plasma was diluted with 4 parts of normal salt solution to 1 of plasma. It was ascertained that the coagulative properties were not interfered with. The observations made after the introduction of this modified plasma showed that much less inflammatory reaction was produced upon the retina, but no improvement with regard to detachment. 
ExPERIMENT 10.- In this case artificial detachment was produced by an injection of 20 per cent. glucose solution behind the retina. Rupture of the retina was not seen. On the third day detachment was quite distinct, but had not increased. On the fifth day detachment had disappeared, and the characteristic pigmentary changes could be seen upon the fundus, over the area where detachment had occurred.

EXPERIMENT 11.-Artificial detachment was in this instance produced by the injection of blood plasma from the horse, which had not been treated to bring about coagulation before being used. No rupture was seen. On the second day marked detachment was present at the lower part of the fundus. On the fourth day marked increase in the extent of detachment was seen. On the eighth day slight increase was seen. On the eleventh day detachment extended over more than half the fundus. On the seventeenth day detachment not extended in area but more prominent. On the twenty-fourth day detachment present to nearly the same extent as on seventeenth day, somewhat altered in shape and apparently slightly decreased. No rise of tension could be detected.

\section{Consideration of Results.}

The frequeut occurrence of detachment in connection with morbid changes in the vitreous is well known, and has frequently been observed clinically, where the products of uveal inflammation or hremorrhage have invaded the vitreous substance. Gross changes in the vitreous, artificially produced-as seen in Experiments 1, 2, and 3 -result in detachment. From my observations I believe Nordenson's description of the mechanism in his second subgroup is the one which applies to my first three experiments. That is to say, the altered vitreous forms abnormal adhesions to the retina posteriorly, and causes detachment by its subsequent contraction.

The general application of the Leber-Nordenson hypothesis, as the explanation of the large majority of cases of idiopathic detachment, is not substantiated by sufficient proof, and there is considerable evidence against it; that is to say, in favour of the theory of diffusion. As Raehlmann has pointed out, the eyes examined post-mortem by Nordenson had been the site of old-standing detachment, and many of the pathological changes found may have been, and probably were, secondary to the occurrence of detachment. The power of absorption exercised by the choroid is very feeble, as compared with that of other vascular structures. This is evidenced by the length of time occupied in the absorption of small quantities of salt solution used in the artificial production of detachment in my experiments. In the case in which a solution of glucose was employed, absorption was much more rapid, the fluid being absorbed in one-fifth of the time taken in the case of salt solution.

On the other hand, the introduction of an albuminous fluid, as in Experiments 8 and 9 , where blood plasma was introduced behind the retina, not only delayed absorption beyond the time taken in the case of salt solution, but caused increase of detachment by augmentation of fluid behind the retina. Whether this increase of post- 
retinal fluid was due to choroidal transudation, or to diffusion from the vitreous, it is difficult to say; but as there was no rupture of the retina seen, and as during the increasing stage of the detachment the ocular tension was not raised, diffusion of vitreous fluid was the more probable explanation.

Scheffels, ${ }^{1}$ in estimating the effect of the galvano-cautery applied to the sclera, found that in rabbits the application caused detachment of the retina. The application of heat to the sclera had probably set up an inflammatory reaction in the choroidal vessels, with consequent transudation of fluid, which from its position behind the retina, and its albuminous nature, would readily set up diffusion from the vitreous, and so cause detachment of the retina.

The theory of diffusion, not only affords an explanation as to how retinal detachment may occur, but is an exceedingly probable explanation, and I believe plays a part in all cases of idiopathic detachment.

Starling, in his paper entitled "The Physiological Factor involved in the Causation of Dropsy," ${ }^{2}$ points out how the fluid contents of the eapillaries is regulated, not only by fluid pressure, but also by osmotic pressure. He proceeds to show how a process of diffusion, taking place between the fluid contents of the lymph spaces and that within the capillaries, is a factor of the highest importance in preserving the continual physiological exchange between substances in solution. Thus the lymph spaces, while they are supplied with lymph which contains a sufficiency of the substances required for nutrition, are not overloaded with fluid.

It is further shown that absorption by blood vessels is much more rapid than is that by the lymphatic vessels. Thus indigo carmine injected into the lymph spaces appears in the urine in five minutes, while it does not appear in the lymph stream for from twenty minutes to two hours. The contents of the capillaries, being richer in proteids than that of the lymph spaces, exert higher osmotic pressure, and thus promote diffusion.

The permeability of the capillary walls to proteid materials differs in various parts of the body in health. Thus, according to Starling, the lymph of the limbs, the filtrate of the comparatively impervious limb capillaries, only contains 2 per cent. to 3 per cent. of proteids; that of the intestinal capillaries 4 per cent. to 6 per cent.; while that from the permeable liver capillaries contains 6 per cent. to 8 per cent.

Experiments performed by Wooldridge (as quoted by Starling) have shown that ligature of the femoral vein, with an injection of fibrinogen, causing artificial thrombosis, is followed by dropsy of the limb. Wooldridge attributes this to the disturbed relationship between

1 Jahresb. ü. d. Leistung. . . . d. Ophth., Tübingen, 1894, S. 415.

${ }^{2}$ Read before the Royal College of Surgeons of Fingland in 1896. 
the blood and the vascular wall. Simple ligature of the femoral vein is not followed by dropsy of the limb, as is well known.

It has been shown by Halliburton ${ }^{1}$ that the fluid effused from the serous membrane in cases of inflammation contains a much higher percentage of proteid material than does fluid effused as a result of pressure.

The fact that various forms of inflammation are accompanied by an excessive transudation of fluid from the blood vessels is sufficiently well known. The facts just referred to, when considered in connection with the physical conditions of the fluids within the eyeball in their relation to the retina, are suggestive.

The transudation from blood vessels, in certain conditions of inflammation, is shown to be not only excessive in amount but abnormally rich in proteids. Such a transudation into the lymph spaces readily upsets the normal balance of osmotic pressure, and thus the lymph spaces remain overloaded with fluid which has an abnormally high percentage of proteids. A short consideration of the relations and nutrition of the retina will show the application of the foregoing to retinal detachment. The vitreous body lies in close relation to the anterior surface of the retina surrounded by the hyaloid membrane.

In composition the vitreous consists almost entirely of water. According to Berzelius, Frerichs, and Lobmeyer, as quoted by Norris and Oliver, ${ }^{2} 98.40$ per cent. to 98.60 per cent. of water is found in the vitreous. The remaining small proportion of solids is composed of salts and extractives, with traces of proteids and nucleo-albumin. Between the retina and the sclerotic there is the choroid. The retinal blood vessels do not penetrate the outer molecular layer. ${ }^{3}$ The stratum of visual cells is nourished by the fluids conveyed by the dense capillary network within the choroid coat, constituting the chorio-capillaris, which lies in close proximity to the outer pigment cells and the underlying percipient elements. The lymphatics of the retina are represented chiefly by the perivascular lymph channels, which surround the veins and capillaries and freely communicate with the subpial lymph spaces of the optic nerve. Injections of the subpial lymph spaces not infrequently pass between the retina and the pigmented epithelium, as well as between the hyaloid membrane and the limitans retina. The extensions of injected Huid have been regarded by Schwalbe as indicating the presence of lymph spaces. ${ }^{4}$

Thus, glancing over the distribution of the blood vessels and lymph channels connected with retinal nutrition, it becomes evident that as in health the layer of hexagonal pigment cells is nourished by the physiological transudation of lymph from the chorio-capillaris, so in

I “Text-Book of Chemical Physiology and Pathology," 1s91, pp. 344-346.

2 "System of Diseases of the Eye," vol. i, p. 364.

"See Schäfer on "Special Senses," p. 55, Quain's "Anatomy," 1897, vol, iii. part 3; and Fuch's "Text-Book of Ophthalmology," 1899 , p. 439.

"Schäfer, Quain's "Anatony," 1897, rol. iii. part 3, p. 56. 
inflammatory conditions of these capillaries, when transudation may become excessive in amount, the neighbourhood of the hexagonal pigment cells will become the site of accumulation, both on account of proximity to the source of lymph, and because of the loose and delicate attachment of the retina at this level. The above reasons may suffice to explain why the plane of cleavage in detachment of the retina is always through the layer of hexagonal pigment cells.

According to the analysis of Scherl, before referred to, the postretinal fluid in some cases of retinal detachment was found to be highly albuminous, in one case reaching 8.9969 per cent. If this is compared with the percentage of proteids normally found in the lymph of other parts of the body, as before noted, it is found to be three times that from the healthy limbs, and even higher than that of the liver, where the blood vessels are normally most permeable to proteids. It is therefore justifiable to regard the percentage of proteid, as found by Scherl's analysis, as probably much greater than that which exists normally in the fluid transuded from the vessels nourishing the retina.

As before noted, serous transudations are more highly albuminous when they result from inflammatory conditions of the affected part, than when produced by pressure. Nordenson has pointed out, that in his investigations he almost invariably found choroidal changes in connection with retinal detachment. The remarkable frequency with which myopia is associated on the one hand with inflammatory disease of the choroid, and on the other with idiopatbic retinal detachment, is worthy of note in this connection.

Without the further enumeration of facts, it seems sufficiently evident that inflammatory processes in the choroid give rise to the albuminous character of the post-retinal fluid, and may account for its primary formation. The increase of this fluid, and the consequent extension of detachment often occurring rapidly and without increase of intraocular tension, can most readily be accounted for by the process of diffusion of the vitreous fluid through the retina, and, in many instances where rupture is not present, cannot be accounted for in any other way.

My experiments, in which various fluids were injected behind the retina, tend-so far as they go - to support the "theory of diffusion." Where albuminous fluid was injected behind the retina-as in Experiment 11 -the detachment which was formed continued to increase for some weeks, no rupture being detected. The introduction of salt solution produced detachment, which attained its maximum either at once or within a few days. The subsequent course was towards healing throughout. In Experiment 5, partial reattachment of the retina was seen with the ophthalmoscope on the twelfth day, and complete reattachment on the twenty-eighth day. 


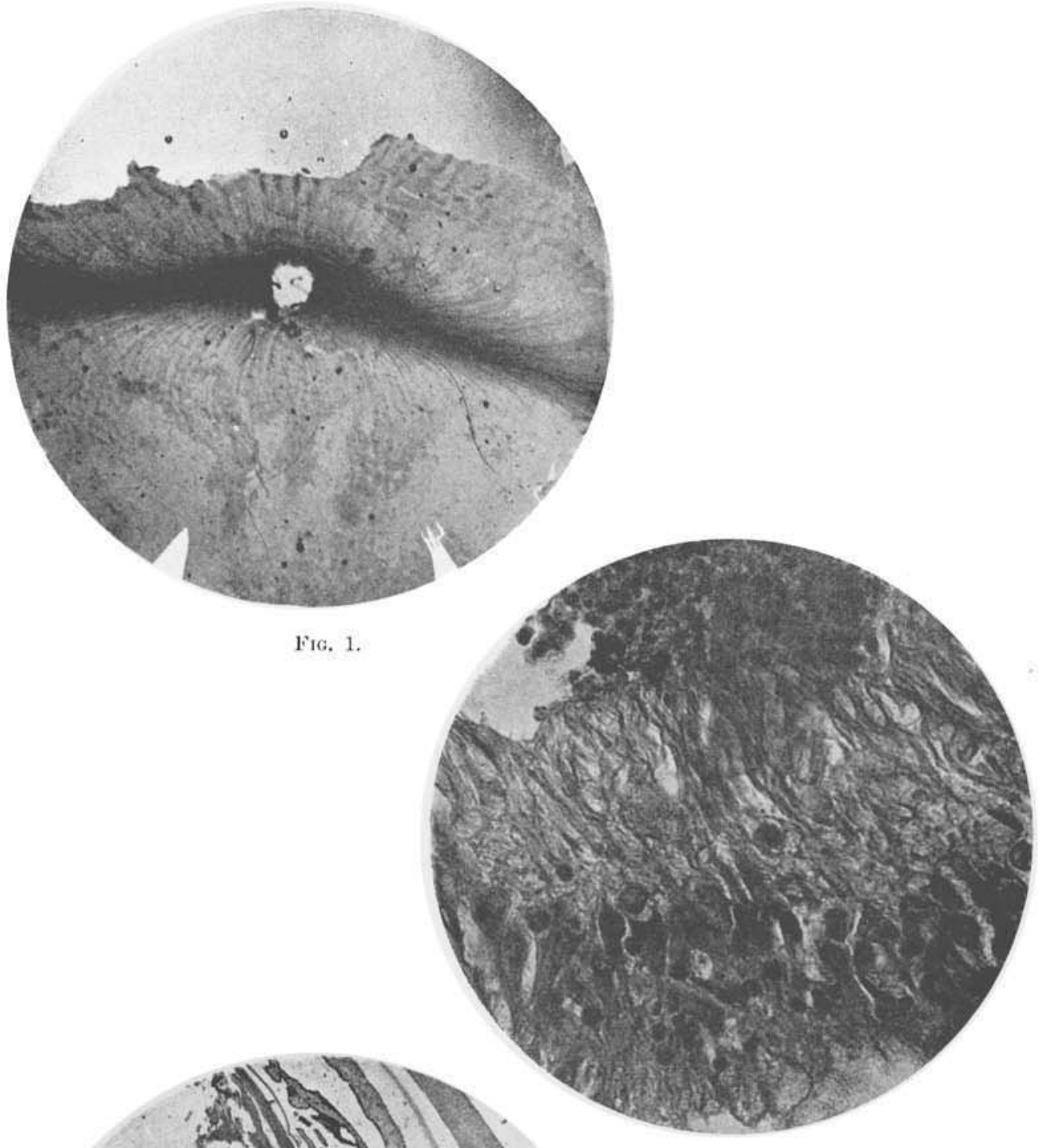

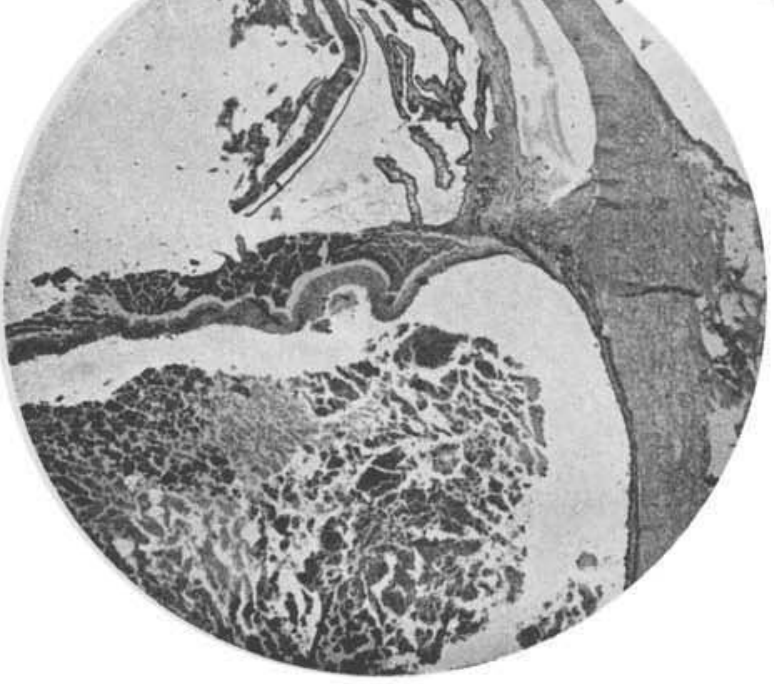

FIG. 2.
Fig. 3.

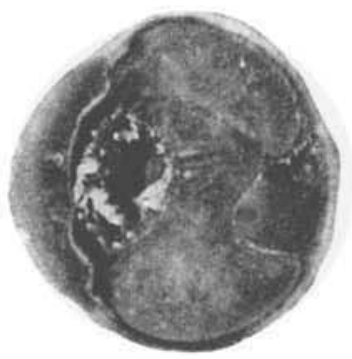

FIG. 4. 
Detachment produced with a 20 per cent. solution of glucose (see Experiment 10) recovered within a week, the diffusibility of the glucose, together with its somewhat irritating effect on the tissues, was probably the cause of its rapid absorption.

The fact that changes in the vitreous often take place in eyes in which retinal detachment subsequently occurs, cannot be taken as any proof that these changes cause detachment of retina. The truth would appear to be that both conditions are brought about by uveal inflammation, and are therefore often found together.

\section{DESCRIPTION OF PLATE XVII.}

F1G. 1.--From a photograph showing the band of opaque nerve fibres in the retina of the rabbit.

Fia. 2.--Inflammatory material which originally lay in the vitreous, and is now behind the detached retina.

FIG. 3,-Degenerated detached retina. Inflammatory material in the vitreous incorporated with degenerated retina.

Fig. 4.-Artificial detachment of the retina produced by injection of fluid as described. 